\title{
New constraints on Higgs-portal scalar dark matter
}

\author{
Huayong Han and Sibo Zheng \\ Department of Physics, Chongqing University, \\ Chongqing 401331, P.R. China \\ E-mail: hhycqu@cqu.edu.cn, sibozheng.zju@gmail.com
}

\begin{abstract}
The simplest Higgs-portal dark matter model, in which a real scalar singlet is added to the standard model, has been comprehensively revisited, by taking into account the constraints from perturbativity, electroweak vacuum stability in the early Universe, dark matter direct detection, and Higgs invisible decay at the LHC. We show that the resonant mass region is totally excluded and the high mass region is reduced to a narrow window $1.1 \mathrm{TeV} \leq m_{s} \leq 2.55 \mathrm{TeV}$, which is slightly reduced to $1.1 \mathrm{TeV} \leq m_{s} \leq 2.0 \mathrm{TeV}$ if the perturbativity is further imposed. This high mass region can be fully detected by the Xenon1T experiment.
\end{abstract}

KeYwords: Beyond Standard Model, Cosmology of Theories beyond the SM

ArXiv EPrint: 1509.01765 


\section{Contents}

1 Introduction 1

2 HDM within perturbative region $\quad 2$

3 Electroweak vacuum stability 3

4 Dark matter phenomenology $\quad 6$

4.1 Relic density 6

$\begin{array}{lll}4.2 & \text { Direct detection } & 7\end{array}$

$\begin{array}{lll}5 & \text { LHC phenomenology } & 8\end{array}$

6 Conclusions and discussions $\quad 9$

\section{Introduction}

The Standard Model (SM) as the effective theory below the electroweak (EW) scale has been established after the discovery of SM Higgs scalar with mass around $125 \mathrm{GeV}[1,2]$ at the Large Hadron Collider (LHC). Unfortunately, there is no viable candidate for a dark matter (DM) in the SM, which implies that an extension beyond the SM is necessary.

Among viable extensions the simplest Higgs-portal dark matter (HDM) [3-7] is the most economic and of special interest from both the DM- and LHC-phenomenology. In this extension, only a real singlet scalar $s$ is added to the SM. With the aid of $Z_{2}$ parity which is needed for the stability of singlet DM, the numner of model parameters is reduced to three. It implies that the minimal model is very predictable. In the subsequent studies on the HDM [8-21], the parameter space is analyzed in terms of experimental constraints as follows.

- Dark matter relic density [22], which shows that the low mass region, resonant mass region, and high mass region are all viable.

- Dark matter direct detection such as Xenon100 [23], Xenon1T [24] and LUX [25] experiments, which are able to effectively detect the high mass region up to $\sim 3 \mathrm{TeV}$.

- Limit on the Higgs invisible decay $h \rightarrow s s$, which can effectively detect the low mass region with $m_{s}$ of order a few $\mathrm{GeV}$.

- Indirect detection such as limit on $\gamma$-ray lines from the Fermi-LAT [27] is rather efficient to constrain the resonant mass region [28-30]. 
However, these constraints are not sufficient, as some possibly strong theoretic ones have been ignored. The object of this paper is to take into account all known experimental and theoretic constraints. Simultaneously, experiments constraints will be updated, if available. In compared with the previous works, e.g., a comprehensive review in [21], our main differences are the following ones.

- The SM EW vacuum suffers from large quantum fluctuations in the early Universe as induced by the inflation, which imposes strong constraint [32-34] on the model parameters. SM vacuum stability must be taken into account to constrain the parameter space. For an earlier attempt, see [35, 36].

- Assume the HDM as an effective theory between the EW and Plank scale, perturbative analysis should be considered seriously, which leads to strong upper bounds on the quartic coupling constant of $s^{2}|H|^{2}$ and the DM self-coupling constant. This effective theory can be applied, e.g., to S-inflation, where the scalar singlet DM is identified as the inflaton [37].

- Very recently, the ATLAS collaboration has reported the latest limit about the Higgs invisible decay width [26] in the low and resonant mass regions. This will be updated in our analysis.

The paper is organized as follows. In section 2, we introduce notation and conventions in the HDM. In this section the constraint on the model parameters from the requirement of perturbativity is studied. In section 3, we discuss the constraint on the model parameters from the SM vacuum stability for $125 \mathrm{GeV}$ Higgs mass. No additional mechanism for stabilizing the SM vacuum stability is employed except the DM scalar.

In section 4 we re-examine the DM phenomenology. In particular, we calculate the $s$ scalar relic density and the spin-independent nucleon-DM scattering cross section by using MicrOMEGAs [38], which are consistent with results in some earlier literature. In section 5, we update the LHC phenomenology about scalar DM, by focusing on the latest limit on the Higgs invisible decay.

Finally, we present our main conclusions in section 6 . We find that the resonant mass region is totally excluded and the high mass region is reduced to a narrow window $1.1 \mathrm{TeV}$ $\leq m_{s} \leq 2.4 \mathrm{TeV}$. If the requirement of perturbativity is further imposed, the allowed mass range is slightly reduced to $1.1 \mathrm{TeV} \leq m_{s} \leq 2.0 \mathrm{TeV}$. In either case the mass window can be fully detected by the Xenon $1 \mathrm{~T}$ experiment.

\section{HDM within perturbative region}

The Lagrangian for the simplest HDM with a $Z_{2}$ parity, under which $s$ is odd and SM particles are even, is given by,

$$
\mathcal{L}=\mathcal{L}_{\mathrm{SM}}+\frac{1}{2}(\partial s)^{2}+V(s, H)
$$

where

$$
V(s, H)=\frac{\lambda}{2}\left(|H|^{2}-\frac{v^{2}}{2}\right)^{2}+\frac{\mu_{s}^{2}}{2} s^{2}+\frac{\lambda_{s}}{2} s^{4}+\frac{\kappa_{s}}{2} s^{2}|H|^{2} .
$$


In eq. (2.2) the first term denotes the Higgs potential with EW scale $v \simeq 246 \mathrm{GeV}$, and the $\lambda_{s}$-term and $\kappa_{s}$-term refers to $s$ self-interaction and Higgs-DM interaction, respectively. Expand the fields as $H=(v+h) / \sqrt{2}$ and $s=\langle s\rangle+s=0+s$ along the vacuum structure for positive $\lambda_{s}$ and $\kappa_{s},{ }^{1}$ one obtains from eq. (2.2),

$$
V(s, h)=\frac{1}{2} m_{s}^{2} s^{2}+\frac{\lambda_{s}}{2} s^{4}+\frac{\kappa_{s} v}{2} s^{2} h+\frac{\kappa_{s}}{4} s^{2} h^{2} .
$$

where $m_{s}=\mu_{s}^{2}+\kappa_{s} v^{2} / 2$. Remarkably, there are only three new parameters in the HDM. It implies that this model can be studied concretely.

In some situations peturbativity should be imposed on the model. This is true when it is assumed to the effective theory below some high energy scale such as Plank mass scale. See, e.g. [37] for an application of such idea to the S-inflation.

In figure 1 we show the upper bounds on the EW values of $\kappa_{s}$ and $\lambda_{s}$ by the requirement of perturbativity

$$
0<\kappa_{s}(\mu)<\sqrt{4 \pi}, \quad 0<\lambda_{s}(\mu)<\sqrt{4 \pi},
$$

for Renormalization Group (RG) scale $\mu$ between the EW and Plank mass scale. We have used two-loop RG equations [41],

$$
\begin{aligned}
& \beta_{\kappa_{s}}=\frac{1}{(4 \pi)^{2}}\left[-\left(\frac{9}{10} g_{1}^{2}+\frac{9}{2} g_{2}^{2}-6 \lambda-6 y_{t}^{2}-12 \lambda_{s}\right) \kappa_{s}+4 \kappa_{s}^{2}\right]+\frac{1}{(4 \pi)^{4}} \cdots \\
& \beta_{\lambda_{s}}=\frac{1}{(4 \pi)^{2}}\left(\kappa_{s}^{2}+36 \lambda_{s}^{2}\right)+\frac{1}{(4 \pi)^{4}} \cdots
\end{aligned}
$$

where $g_{i}$ refer to the SM gauge coupling, and $y_{t}$ denotes the top Yukawa coupling. As expected from eq. (2.5), the correlation between the upper bounds on $\kappa_{s}$ and $\lambda_{s}$ is mildly sensitive to $s$ scalar mass, but strongly to each other. Our scan shows that the critical values are given by $\kappa_{s}<0.6$ and $\lambda_{s}<0.131$.

Of course, these bounds can be relaxed when the high energy scale above which the model is not effective is smaller than our reference value.

\section{Electroweak vacuum stability}

It was shown in [31] that the EW vacuum is metastable for the observed Higgs mass $m_{h} \simeq 125 \mathrm{GeV}$, as seen from the RG equation for $\lambda$. Since the sign of beta function $\beta_{\lambda}$ is always negative in the SM, $\lambda$ becomes negative above some critical RG scale $\mu_{*} \cdot \mu_{*}$ is approximately equal to the value $h_{*}$, at which the Higgs potential is maximal. For the central values of top quark mass $m_{t}=173.2 \mathrm{GeV}$ and structure constant of QCD gauge coupling $\alpha=0.1184, h_{*} \simeq 10^{9}-10^{10} \mathrm{GeV}$ at the two-loop level. The situation can be improved to $h_{*} \simeq 10^{19} \mathrm{GeV}$ for lighter $m_{t}$ within $1 \sigma$ uncertainty [42]. So, in the most of SM parameter space EW vacuum is only metastable.

\footnotetext{
${ }^{1}$ The vacuum is given by $\langle H\rangle=v / \sqrt{2}$ and $\langle s\rangle=0$ for both positive $\lambda_{s}$ and $\kappa_{s}$, which may be violated when both of them are negative. However, as we will shown in section 3 , neither negative $\kappa_{s}$ and nor negative $\lambda_{s}$ are favored by the EW vacuum stability. In this paper we choose both positive $\lambda_{s}$ and $\kappa_{s}$ for our discussion.
} 


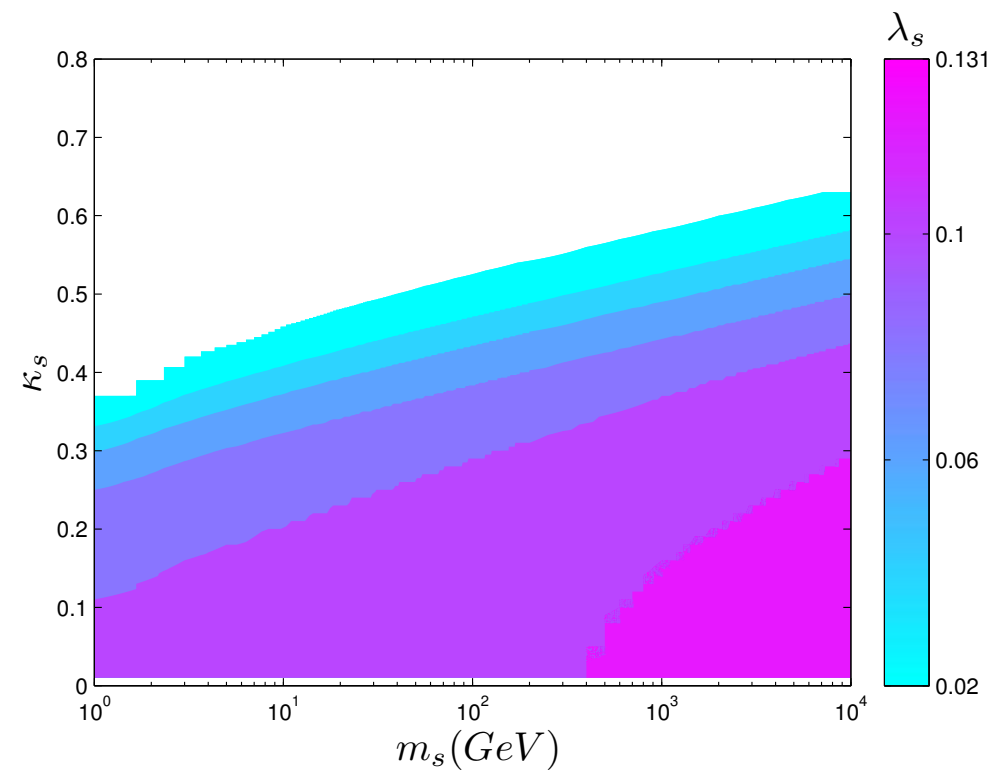

Figure 1. Upper bounds on the EW values of $\kappa_{s}$ and $\lambda_{s}$ from the requirement of perturbativity below the Plank scale.

For EW vacuum being metastable, it suffers from large quantum fluctuation in the early Universe, especially during and after inflation, which leads to strong theoretic constraint on the model parameters.

Let us firstly discuss the survival probability of EW vacuum during inflation. Denote $P(h, t)$ the probability for Higgs field with the value $h$ at time $t$, which can be determined via solving the Fokker-Plank equation [32-34],

$$
\frac{\partial P}{\partial t}=\frac{\partial}{\partial h}\left[\frac{H_{c}^{3}}{8 \pi^{2}} \frac{\partial P}{\partial h}+\frac{V^{\prime}(h)}{3 H_{c}} P\right] .
$$

Here $H_{c}$ refers to the Hubble parameter, ${ }^{2}$ which is approximate constant during inflation. $V^{\prime}$ denotes derivative of Higgs potential over Higgs field. For the case $h<h_{*}$ the quantum fluctuation- $H_{c}^{3}$ term dominates the classical one-the last term in eq. (3.1). If one ignores this classical term, eq. (3.1) can be easily solved in terms of separating variants and resonable boundary conditions. See $[33,34]$ for details. Then, the probability for staying at the domain $h>h_{*}$ other than EW vacuum at the end of inflation is given by,

$$
P\left(|h|>h_{*}\right)=1-\int_{-h_{*}}^{h_{*}} P\left(h, \frac{N}{H_{c}}\right) d h
$$

where $N \simeq 55-60$ is the number of e-folds.

If the probability for staying at the metastable EW vacuum is not zero as required, $P\left(|h|>h_{*}\right)$ should be smaller than $e^{-3 N}$, which gives rise to,

$$
h_{*}>\sqrt{\frac{3}{2}} \frac{N}{\pi} H_{c} \simeq 25 H_{c} \simeq 2.5 \times 10^{17} \mathrm{GeV} .
$$

\footnotetext{
${ }^{2}$ The magnitude of Hubble constant is directly related to the scalar-to-tensor ratio $r$, with $H_{c} \simeq 1 \times$ $10^{16} \times(r / 0.01)^{1 / 4} \mathrm{GeV}$. We take $H_{c}=10^{16} \mathrm{GeV}$ as the reference point, which is a good approximation unless $r$ is extremely small.
} 


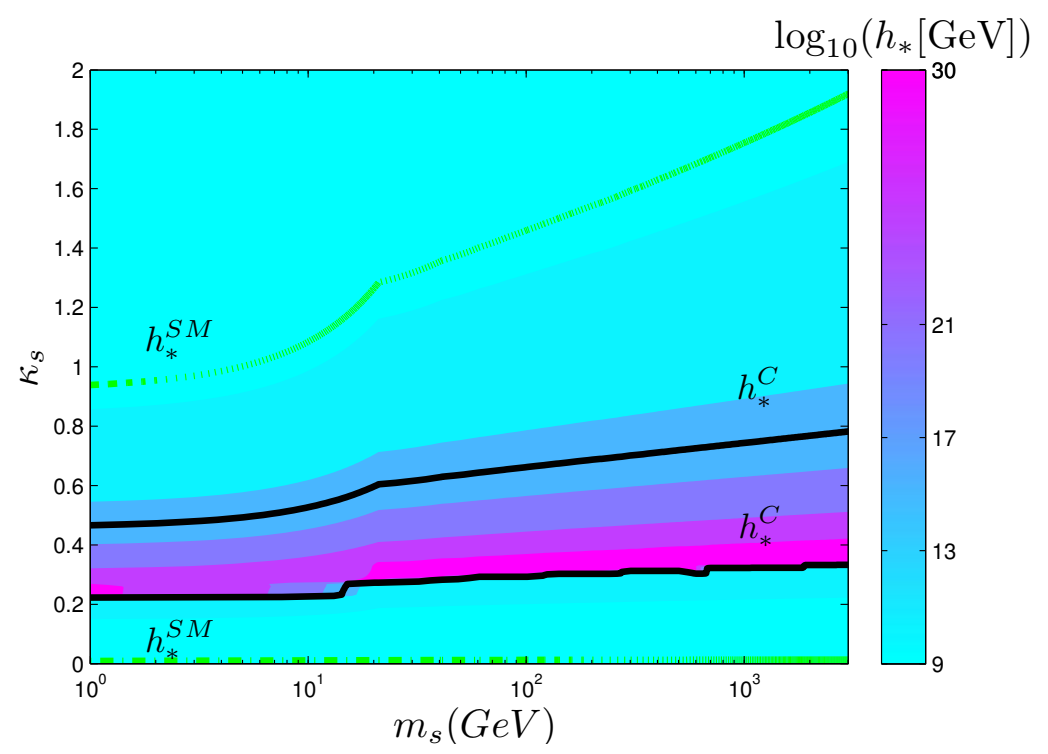

Figure 2. The value of $h_{*}$ at which the Higgs potential is maximal in the parameter space of $\kappa_{s}-m_{s}$. The green curves correspond to the case of SM. The black curves show the critical value as required by EW vacuum stability. It clearly shows that the value of $h_{*}$ can be uplifed into the region surrounded by the two black curves.

Obviously, this condition cannot be satisfied in the most of parameter space of SM. The constraint in eq. (3.3) can be relaxed in the context of SM by introducing a new interation $\xi_{H} \mathcal{R}^{2}|H|^{2}[34,43]$ between the Higgs and Ricci scalar $\mathcal{R}$.

With no need of parameter $\xi_{H}$, this reduction can be alternatively realized in the HDM. It is easy to understand in terms of the new contribution to beta function $\beta_{\lambda}$, which reads at the two-loop level,

$$
\delta \beta_{\lambda}=\beta_{\lambda}^{(\mathrm{HDM})}-\beta_{\lambda}^{(\mathrm{SM})}=\frac{\kappa_{s}^{2}}{(4 \pi)^{2}}+\frac{1}{(4 \pi)^{4}}\left(-5 \lambda \kappa_{s}^{2}-4 \kappa_{s}^{3}\right) .
$$

The value of $h_{*}$ can be uplifted for small $\kappa_{s}$, as the sign of the new contribution in eq. (3.4) can be positive in this region. In contrast, $h_{*}$ may be reduced in the region of large $\kappa_{s}$, where the sign of the new contribution in eq. (3.4) is negative. The green curve in figure 2 clearly shows the critical value $\kappa_{s}^{c} \sim 0.9 .^{3}$ Below this critical value $h_{*}$ can be larger than $\mathrm{SM}$ value $h_{*}^{\mathrm{SM}}$. More interesting, as shown by the red region corresponding to the range $\kappa_{s} \simeq 0.25-0.8$, the required value $h_{*}^{c}=2.5 \times 10^{17} \mathrm{GeV}$ in eq. (3.3) can be satisfied.

The effect on the vacuum stability of the singlet scalar potential due to quantum fluctuations is more easily understood, in comparison with the Higgs potential. Since $\langle s\rangle=0$ is the true minimal of singlet scalar potential, quantum fluctuation with magnitude of order $\delta s \sim H_{c}$ may push $s$ towards to an unstable vacuum from the origin. But it rapidly returns to the origin vacuum through classical tunning process. This understanding holds as long as $\lambda_{s}$ is always positive between the EW and Plank scale.

\footnotetext{
${ }^{3}$ For negative $\kappa_{s},\left|\kappa_{s}^{c}\right|$ is larger in comparison with positive $\kappa_{s}$. However, its magnitude is more seriously bounded above by the analysis of perturbativity.
} 


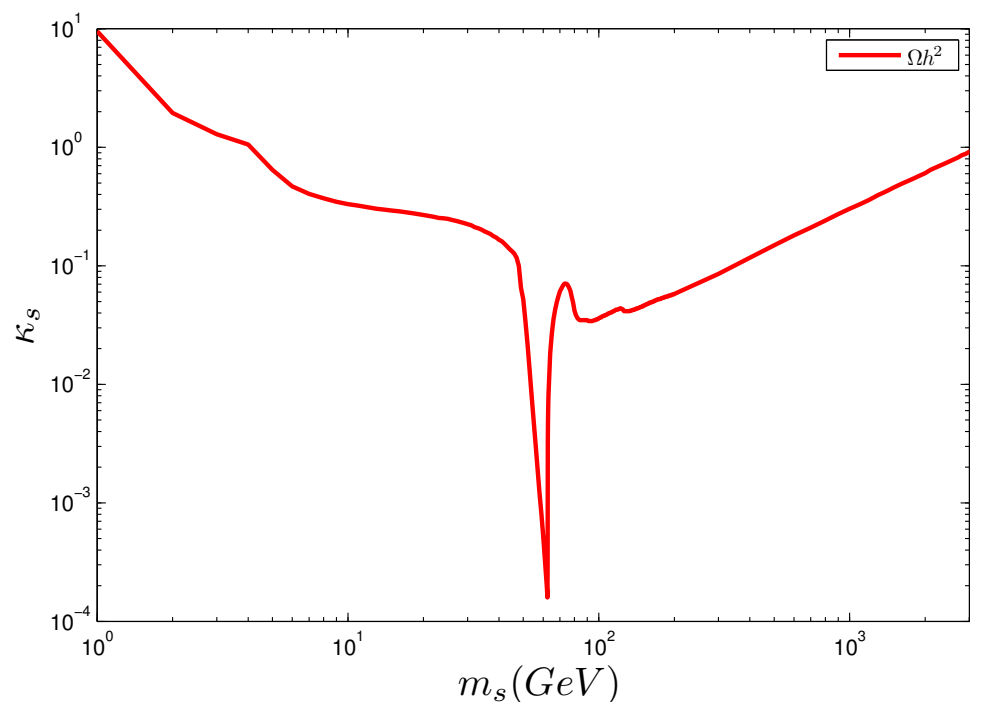

Figure 3. Contours of $s$ scalar relic density $\Omega_{s} h^{2}$ as required by the DM projected to the twoparameter plane of $\left(m_{s}, \kappa_{s}\right)$.

Once inflation ends, (p)reheating begins immediately. The quantum effect on the EW vacuum during (p)reheating can be studied in terms of the equations of motion for $s$ and Higgs in principle. Unfortunately, a concrete constraint on the model parameters in HDM can not be derived without knowledge of reheating temperature $T_{\text {re }}$, which is an important physical quantity involved [34]. To determine $T_{\mathrm{re}}$ interactions between the inflaton scalar and SM fields should be given explicitly.

\section{Dark matter phenomenology}

In this section we firstly consider the constraints arising from the DM relic density and direct detection limits at the LUX and Xenon100 experiments. Then we discuss the prospect at the Xenon1T experiments.

\subsection{Relic density}

The Plank and WMAP 9-year data have measured the DM relic density in high precision [22],

$$
\Omega_{\mathrm{DM}} h^{2}=0.1199 \pm 0.0027
$$

With the assumption that the relic density of $s$ scalar is totally produced by the thermal freeze-out process, we have

$$
\Omega_{s} h^{2} \sim 0.1 \mathrm{pb} /\left\langle\sigma_{\mathrm{ann}} v\right\rangle,
$$

where $\sigma_{\text {ann }}$ is the total annihilation cross-section for $s$ and $v$ is the relative velocity.

The $s s$ annihilation mainly proceeds through Higgs-mediated process in the $s$ channel. Sub-dominant process include $s$ exchange in the $t$ channel and annihilation into $h h$. So, $\sigma_{\text {ann }}$ is sensitive to the model parameters $\kappa_{s}$ and $m_{s}$. 


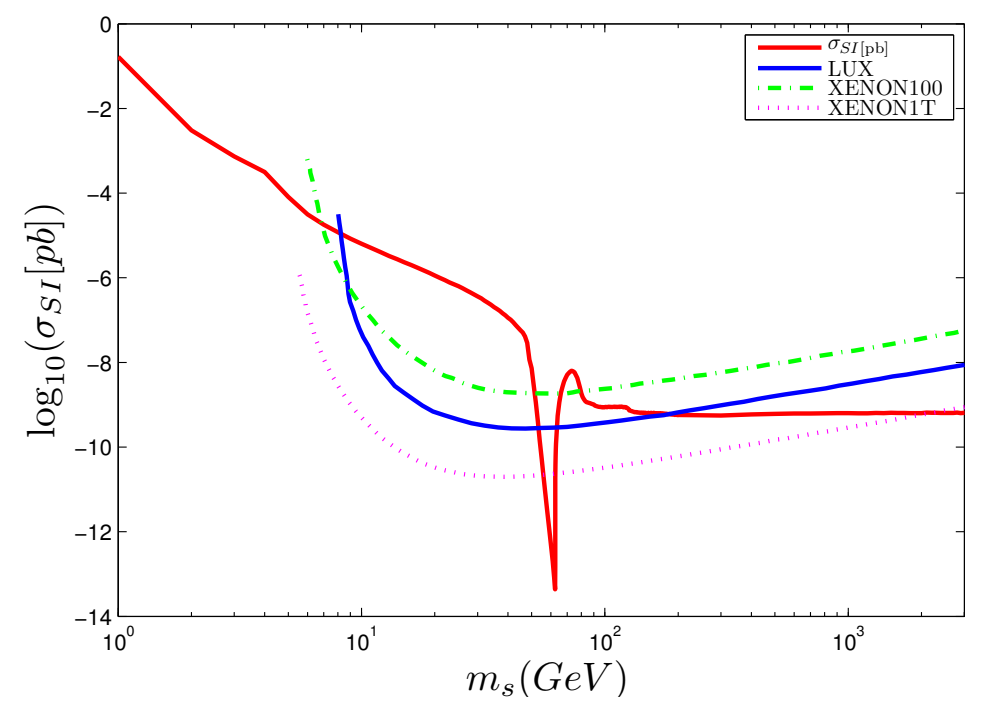

Figure 4. Spin-independent nucleon-DM scattering cross section as function of $m_{s}$. Various experimental limits are also shown for comparison.

Instead of analytic method we use the code MicrOMEGAs [38] to calculate the $s$ scalar relic density $\Omega_{s} h^{2}$, with the Feynman rules generated by the package LanHEP 3.2.0 [39]. In figure 3 we project the contour of relic density $\Omega_{s} h^{2}$ as required by eq. (4.1) to the twoparameter plane of $\left(m_{s}, \kappa_{s}\right)$. As expected the required value of $\kappa_{s}$ is minimal for $m_{s}$ near $m_{h} / 2$, because near this mass region the annihilation cross section is resonantly enhanced. Plot similar to figure 3 is also shown, e.g., in [21]. ${ }^{4}$

Figure 3 indicates that a wide mass range for $s$ scalar is viable. However, it also shows that $\kappa_{s}$ bigger than 0.1 is required when $m_{s}$ is far away from the resonant mass region. For example, large $\kappa_{s} \geq 0.5$ is needed for $m_{s}$ above $3 \mathrm{TeV}$, which is not favored neither by the EW vacuum stability nor the perturbative analysis. In the next subsection, we discuss the direct detection in the allowed parameter space.

\subsection{Direct detection}

The spin-independent nucleon-DM scattering cross section is given by,

$$
\sigma_{\mathrm{SI}}=\frac{\kappa_{s}^{2} f_{N}^{2} \mu^{2} m_{N}^{2}}{4 \pi m_{h}^{4} m_{s}^{2}}
$$

where $m_{N}$ is the nucleon mass, $\mu=m_{s} m_{N} /\left(m_{s}+m_{N}\right)$ is the DM-nucleon reduced mass, and $f_{N} \sim 0.3[21]$ is the hadron matrix element.

The current best limit on $\sigma_{\mathrm{SI}}$ comes from the Xenon100 [23] and LUX [25] experiments. In figure 4 we plot the spin-independent nucleon-DM scattering cross section as function

\footnotetext{
${ }^{4}$ Although these plots match very well, we would like to mention that contributions to $\sigma_{\text {ann }}$ due to threeand four-body final states or QCD corrections are not included in MicrOMEGAs, with which the shape around $W$ boson mass is smoother [40]. We thank the referee for reminding us this point. Since DM mass region near $80 \mathrm{GeV}$ has been excluded by the LUX experiment, these corrections are expected to not affect our main results in section6.
} 


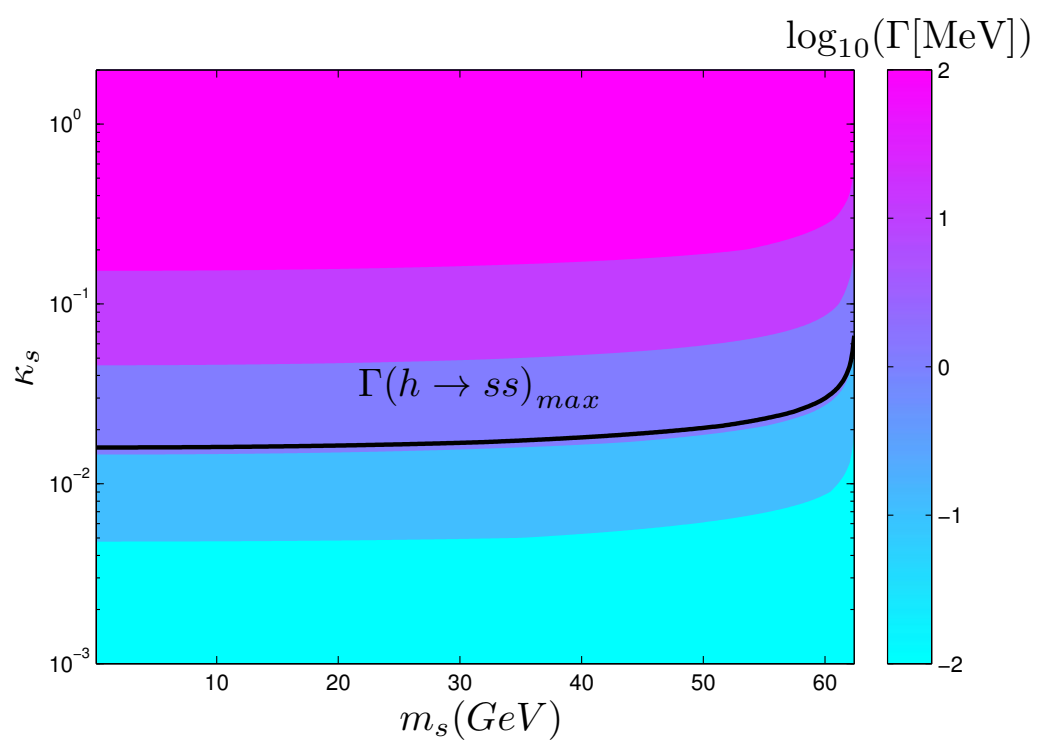

Figure 5. Contours of invisible decay width $\Gamma(h \rightarrow s s)$ in the two-parameter plane of $m_{s}-\kappa_{s}$. The contour in black denotes the latest upper bound in eq. (5.1). Region above this line is excluded.

of $m_{s}$, with various experimental limits for comparison. The red curve corresponds to the DM relic density, which indicates that there are three viable regions,

$$
\begin{aligned}
& \text { low mass region : } 1 \mathrm{GeV} \leq m_{s} \leq 6 \mathrm{GeV}, \\
& \text { resonant mass region : } 56 \mathrm{GeV} \leq m_{s} \leq 66 \mathrm{GeV}, \\
& \text { high mass region : } m_{s} \geq 185 \mathrm{GeV} .
\end{aligned}
$$

By combing the constraint from SM vacuum stability, one finds that

- The resonant mass region is totally excluded, which is more powerful than the indirect constraint from the Fermi-LAT [27].

- The high mass region is further reduced to more narrow mass window, which is the subject of Xenon 1T [24] experiment for $m_{s}$ below $4 \mathrm{TeV}$.

- The low mass region can be consistent with the constraint from SM vacuum stability. However, we will show in sectionV that the low mass region is excluded by the Higgs invisible decay $h \rightarrow s s$ at the LHC experiment (see also some literature in refs. [8]$[18])$.

\section{$5 \quad$ LHC phenomenology}

Since the scalar singlet only couples to the Higgs directly, the signal searches for this scalar at the LHC mainly focus on the Higgs invisible decay $h \rightarrow s s$ in the low mass region and resonant mass region with $m_{s}<m_{h} / 2$. Note that for scalar singlet DM there is no mixing effect ${ }^{5}$ between $s$ and $h$, as clearly shown in eq. (2.1) and eq. (2.2).

\footnotetext{
${ }^{5}$ If $s$ scalar is not DM, one may discuss its phenomenology by introducing mixing effects. For a recent discussion, see [44].
} 


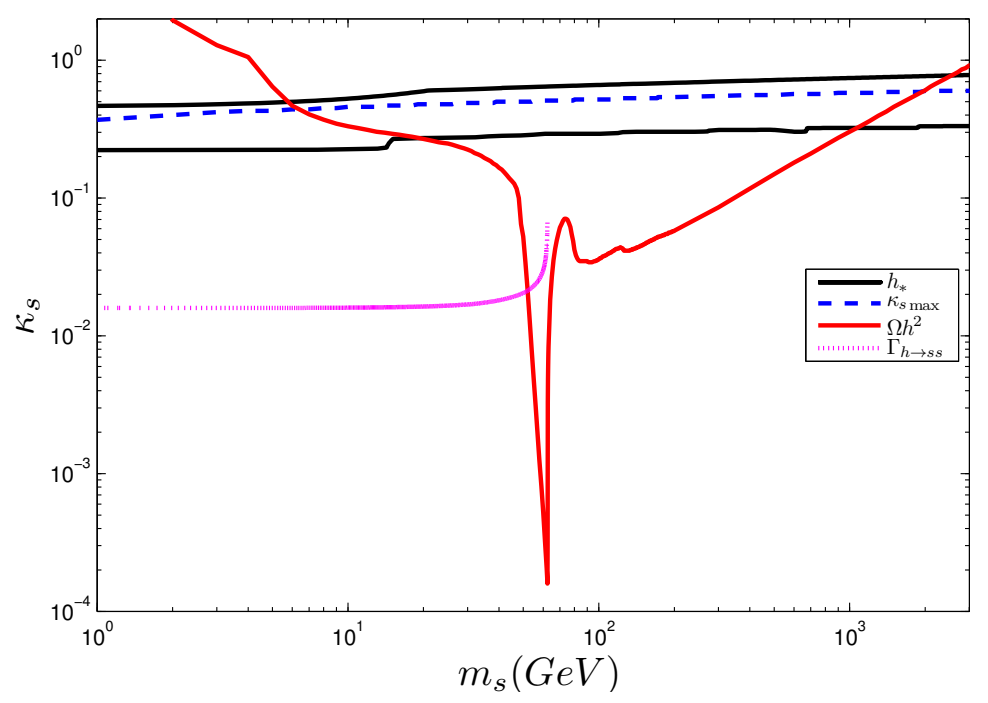

Figure 6. Mass regions consistent with various constraints. In the light of the SM vacuum stability, only mass region $1.1 \mathrm{TeV} \leq m_{s} \leq 2.5 \mathrm{TeV}$ is viable, and it slightly reduces to $1.1 \mathrm{TeV}$ $\leq m_{s} \leq 2.0 \mathrm{TeV}$ by the additional requirement of perturbativity. For the explanation about $h_{*}$ and $\kappa_{s \max }$, see previous discussions.

Very recently, the ATLAS Collaboration has reported the latest data about the upper bound on the Higgs invisible decay width [26],

$$
\Gamma_{\text {inv }} \leq 0.29 \Gamma_{\mathrm{SM}}
$$

which is obviously stronger than what was used in some earlier literature. An update is thus meaningful. In eq. (5.1) $\Gamma_{\mathrm{SM}} \simeq 4.15 \mathrm{MeV}$ for $125 \mathrm{GeV}$ Higgs mass, and the invisible decay width $\Gamma_{\text {inv }}$ is given by,

$$
\Gamma(h \rightarrow s s)=\frac{\kappa_{s}^{2} v^{2}}{32 \pi m_{h}} \sqrt{1-\frac{4 m_{s}^{2}}{m_{h}^{2}}} .
$$

We show the decay width $\Gamma(h \rightarrow s s)$ in figure 5 . The contour in black denotes the latest upper bound in eq. (5.1), and region above it is excluded. In particular, this bound excludes the whole low mass region and the resonant mass region with $m_{s} \leq 62.5 \mathrm{GeV}$ in eq. (4.3). In contrast, the high mass region is less constrained at the LHC in compared with the low mass region.

\section{Conclusions and discussions}

In this paper we have reconsidered the simplest HDM. By combining the constraints arising from DM relic density, direct detection limit on spin-independent nucleon-DM scattering cross section from the LUX, and limit on the Higgs invisible decay from the LHC, we have obtained two viable mass regions (see figure 6):

resonant mass region : $62.5 \mathrm{GeV} \leq m_{s} \leq 66 \mathrm{GeV}$,

high mass region $: m_{s} \geq 185 \mathrm{GeV}$. 
We also show that if the constraint from the SM vacuum stability is imposed, we arrive at the main conclusion- the resonant mass region is totally excluded and the high mass region in eq. (6.1) is reduced to a narrow window,

$$
\text { high mass region : } 1.1 \mathrm{TeV} \leq m_{s} \leq 2.55 \mathrm{TeV} \text {, }
$$

as shown in figure 6 . If one further imposes the constraint due to perturbativity, the mass range in eq. (6.2) is slightly reduced to,

$$
\text { high mass region : } 1.1 \mathrm{TeV} \leq m_{s} \leq 2.0 \mathrm{TeV} \text {. }
$$

Fortunately, as shown in figure 4 the mass region in eq. (6.3) can be totally detected by the Xenon $1 \mathrm{~T}$ experiment in the near further. Similarly to Xenon $1 \mathrm{~T}$ experiment, this high mass region can be also directly detected at colliders in principle. As a result of small production cross section of order less than $1 \mathrm{fb}$ for the $s$ scalar at the 14-TeV LHC [19], a $5 \sigma$ discovery acquires luminosity at least of order $\mathcal{O}(10) a b^{-1}$.

Independently, effects on large scale structure induced by DM self-interaction is a new window for the detection. In compared with the abelian vector- or fermion-like DM, $s$ scalar with quartic self-interaction is very distinctive. DM self-interaction has been used to interpret [47] the observations on four galaxies in the core of galaxy cluster Abell 3827 [46]. If the new observations indeed arise from the DM self-interaction other than astrophysical artifact, the high mass region is highly constrained, see, e.g., for recent discussion [45].

In variety of contexts the high mass region in eq. (6.2) may be modified. For example, it can be relaxed if one employs additional mechanism to stabilize the SM vacuum by introducing more new parameters, such as the non-minimal interaction between the Higgs and Ricci scalar with non-negligible coupling constant. In contrast, this high mass region may be alternatively strengthened if one assumes [21] that the DM relic abundance is partially other than totally saturated by the scalar singlet. In this case scalar $s$ may play a positive role in the electroweak baryogenesis [40].

\section{Acknowledgments}

HH would like to thank Felix Kahlhoefer for suggesting the use of code LanHEP. This work is supported in part by the Natural Science Foundation of China under grant No.11247031 and 11405015 .

Open Access. This article is distributed under the terms of the Creative Commons Attribution License (CC-BY 4.0), which permits any use, distribution and reproduction in any medium, provided the original author(s) and source are credited.

\section{References}

[1] ATLAS collaboration, Combined search for the Standard Model Higgs boson using up to $4.9 \mathrm{fb}^{-1}$ of pp collision data at $\sqrt{s}=7 \mathrm{TeV}$ with the ATLAS detector at the LHC, Phys. Lett. B 710 (2012) 49 [arXiv: 1202.1408] [INSPIRE]. 
[2] CMS collaboration, Combined results of searches for the standard model Higgs boson in pp collisions at $\sqrt{s}=7 \mathrm{TeV}$, Phys. Lett. B 710 (2012) 26 [arXiv:1202.1488] [InSPIRE].

[3] V. Silveira and A. Zee, Scalar phantoms, Phys. Lett. B 161 (1985) 136 [InSPIRE].

[4] J. McDonald, Gauge singlet scalars as cold dark matter, Phys. Rev. D 50 (1994) 3637 [hep-ph/0702143] [INSPIRE].

[5] J. McDonald, Thermally generated gauge singlet scalars as selfinteracting dark matter, Phys. Rev. Lett. 88 (2002) 091304 [hep-ph/0106249] [INSPIRE].

[6] M.C. Bento, O. Bertolami, R. Rosenfeld and L. Teodoro, Selfinteracting dark matter and invisibly decaying Higgs, Phys. Rev. D 62 (2000) 041302 [astro-ph/0003350] [INSPIRE].

[7] C.P. Burgess, M. Pospelov and T. ter Veldhuis, The minimal model of nonbaryonic dark matter: a singlet scalar, Nucl. Phys. B 619 (2001) 709 [hep-ph/0011335] [INSPIRE].

[8] H. Davoudiasl, R. Kitano, T. Li and H. Murayama, The new minimal standard model, Phys. Lett. B 609 (2005) 117 [hep-ph/0405097] [INSPIRE].

[9] A. Kusenko, Sterile neutrinos, dark matter and the pulsar velocities in models with a Higgs singlet, Phys. Rev. Lett. 97 (2006) 241301 [hep-ph/0609081] [INSPIRE].

[10] D. O'Connell, M.J. Ramsey-Musolf and M.B. Wise, Minimal extension of the standard model scalar sector, Phys. Rev. D 75 (2007) 037701 [hep-ph/0611014] [INSPIRE].

[11] V. Barger, P. Langacker, M. McCaskey, M.J. Ramsey-Musolf and G. Shaughnessy, LHC phenomenology of an extended standard model with a real scalar singlet, Phys. Rev. D 77 (2008) 035005 [arXiv:0706.4311] [INSPIRE].

[12] H. Sung Cheon, S.K. Kang and C.S. Kim, Low scale leptogenesis and dark matter candidates in an extended seesaw model, JCAP 05 (2008) 004 [Erratum ibid. 03 (2011) E01] [arXiv:0710.2416] [INSPIRE].

[13] X.-G. He, T. Li, X.-Q. Li, J. Tandean and H.-C. Tsai, Constraints on scalar dark matter from direct experimental searches, Phys. Rev. D 79 (2009) 023521 [arXiv:0811.0658] [INSPIRE].

[14] R.N. Lerner and J. McDonald, Gauge singlet scalar as inflaton and thermal relic dark matter, Phys. Rev. D 80 (2009) 123507 [arXiv:0909.0520] [INSPIRE].

[15] M. Farina, D. Pappadopulo and A. Strumia, CDMS stands for constrained dark matter singlet, Phys. Lett. B 688 (2010) 329 [arXiv:0912.5038] [INSPIRE].

[16] W.-L. Guo and Y.-L. Wu, The real singlet scalar dark matter model, JHEP 10 (2010) 083 [arXiv: 1006.2518 ] [INSPIRE].

[17] S. Profumo, L. Ubaldi and C. Wainwright, Singlet scalar dark matter: monochromatic gamma rays and metastable vacua, Phys. Rev. D 82 (2010) 123514 [arXiv:1009.5377] [InSPIRE].

[18] A. Biswas and D. Majumdar, The real gauge singlet scalar extension of standard model: a possible candidate of cold dark matter, Pramana 80 (2013) 539 [arXiv:1102.3024] [INSPIRE].

[19] A. Djouadi, O. Lebedev, Y. Mambrini and J. Quevillon, Implications of LHC searches for Higgs-portal dark matter, Phys. Lett. B 709 (2012) 65 [arXiv:1112.3299] [INSPIRE].

[20] A. Djouadi, A. Falkowski, Y. Mambrini and J. Quevillon, Direct detection of Higgs-portal dark matter at the LHC, Eur. Phys. J. C 73 (2013) 2455 [arXiv:1205.3169] [INSPIRE].

[21] J.M. Cline, K. Kainulainen, P. Scott and C. Weniger, Update on scalar singlet dark matter, Phys. Rev. D 88 (2013) 055025 [arXiv: 1306.4710] [INSPIRE]. 
[22] Planck collaboration, P.A.R. Ade et al., Planck 2013 results. XVI. Cosmological parameters, Astron. Astrophys. 571 (2014) A16 [arXiv:1303.5076] [INSPIRE].

[23] XENON100 collaboration, E. Aprile et al., Dark matter results from 225 live days of XENON100 data, Phys. Rev. Lett. 109 (2012) 181301 [arXiv:1207.5988] [InSPIRE].

[24] XENON1T collaboration, E. Aprile, The XENON1T dark matter search experiment, Springer Proc. Phys. 148 (2013) 93 [arXiv:1206.6288] [InSPIRE].

[25] LUX collaboration, D.S. Akerib et al., First results from the LUX dark matter experiment at the Sanford Underground Research Facility, Phys. Rev. Lett. 112 (2014) 091303 [arXiv: 1310.8214] [INSPIRE].

[26] ATLAS collaboration, Search for an invisibly decaying higgs boson produced via vector boson fusion in pp collisions at $\sqrt{s}=8 \mathrm{TeV}$ using the ATLAS detector at the LHC, ATLAS-CONF-2015-004 (2015).

[27] Fermi-LAT collaboration, M. Ackermann et al., Search for gamma-ray spectral lines with the Fermi large area telescope and dark matter implications, Phys. Rev. D 88 (2013) 082002 [arXiv: 1305.5597] [INSPIRE].

[28] L. Feng, S. Profumo and L. Ubaldi, Closing in on singlet scalar dark matter: LUX, invisible Higgs decays and gamma-ray lines, JHEP 03 (2015) 045 [arXiv:1412.1105] [INSPIRE].

[29] M. Duerr, P. Fileviez Perez and J. Smirnov, Scalar singlet dark matter and gamma lines, Phys. Lett. B 751 (2015) 119 [arXiv:1508.04418] [INSPIRE].

[30] C.E. Yaguna, Gamma rays from the annihilation of singlet scalar dark matter, JCAP 03 (2009) 003 [arXiv:0810.4267] [INSPIRE].

[31] D. Buttazzo et al., Investigating the near-criticality of the Higgs boson, JHEP 12 (2013) 089 [arXiv: 1307.3536] [INSPIRE].

[32] A. Kobakhidze and A. Spencer-Smith, Electroweak vacuum (in)stability in an inflationary universe, Phys. Lett. B 722 (2013) 130 [arXiv:1301.2846] [INSPIRE].

[33] A. Hook, J. Kearney, B. Shakya and K.M. Zurek, Probable or improbable universe? Correlating electroweak vacuum instability with the scale of inflation, JHEP 01 (2015) 061 [arXiv: 1404.5953] [INSPIRE].

[34] J.R. Espinosa et al., The cosmological Higgstory of the vacuum instability, JHEP 09 (2015) 174 [arXiv: 1505. 04825] [INSPIRE].

[35] N. Khan and S. Rakshit, Study of electroweak vacuum metastability with a singlet scalar dark matter, Phys. Rev. D 90 (2014) 113008 [arXiv:1407.6015] [INSPIRE].

[36] M. Kadastik, K. Kannike, A. Racioppi and M. Raidal, Implications of the $125 \mathrm{GeV}$ Higgs boson for scalar dark matter and for the CMSSM phenomenology, JHEP 05 (2012) 061 [arXiv: 1112.3647] [INSPIRE].

[37] F. Kahlhoefer and J. McDonald, WIMP dark matter and unitarity-conserving inflation via a gauge singlet scalar, JCAP 11 (2015) 015 [arXiv: 1507.03600] [INSPIRE].

[38] G. Bélanger, F. Boudjema, A. Pukhov and A. Semenov, MicrOMEGAs4.1: two dark matter candidates, Comput. Phys. Commun. 192 (2015) 322 [arXiv:1407.6129] [InSPIRE].

[39] A. Semenov, LanHEP - A package for automatic generation of Feynman rules from the Lagrangian. Updated version 3.2, arXiv:1412.5016 [INSPIRE]. 
[40] J.M. Cline and K. Kainulainen, Electroweak baryogenesis and dark matter from a singlet Higgs, JCAP 01 (2013) 012 [arXiv:1210.4196] [INSPIRE].

[41] C. Cheung, M. Papucci and K.M. Zurek, Higgs and dark matter hints of an oasis in the desert, JHEP 07 (2012) 105 [arXiv: 1203.5106] [INSPIRE].

[42] S. Zheng, Can Higgs inflation be saved with high-scale supersymmetry?, Eur. Phys. J. C 75 (2015) 489 [arXiv: 1504.08093] [INSPIRE].

[43] J.R. Espinosa, G.F. Giudice and A. Riotto, Cosmological implications of the Higgs mass measurement, JCAP 05 (2008) 002 [arXiv:0710.2484] [INSPIRE].

[44] S. Zheng, Discovery of scalar mixed with SM Higgs via diboson excess at the LHC, arXiv:1508.06014 [INSPIRE].

[45] R. Campbell, S. Godfrey, H.E. Logan, A.D. Peterson and A. Poulin, Implications of the observation of dark matter self-interactions for singlet scalar dark matter, Phys. Rev. D 92 (2015) 055031 [arXiv: 1505. 01793] [INSPIRE].

[46] R. Massey et al., The behaviour of dark matter associated with four bright cluster galaxies in the 10 kpc core of Abell 382\%, Mon. Not. Roy. Astron. Soc. 449 (2015) 3393 [arXiv: 1504.03388] [INSPIRE].

[47] F. Kahlhoefer, K. Schmidt-Hoberg, J. Kummer and S. Sarkar, On the interpretation of dark matter self-interactions in Abell 3827, Mon. Not. Roy. Astron. Soc. 452 (2015) L54 [arXiv: 1504.06576] [INSPIRE]. 